\title{
A Randomized, Double Blind, Vehicle Controlled, Parallel, Phase li Study to Evaluate Efficacy and Safety of Bac in Patients With Alzheimer's Disease or Vascular Dementia
}

\author{
Stephen Thein \\ Pacific Research Network \\ George Konis \\ Woodland International Research Group \\ Dennis Michael Lacey \\ NeuroTrials, Inc \\ Fayz Hudefi \\ Woodland Research Northwest \\ Joel Vandersluis \\ Neurology Diagnostic, Inc. \\ Michelle Papka \\ The Cognitive and Research Center of NJ \\ Sanjiv Sharma \\ Advanced Memory Research Institute of NJ \\ Corey G. Anderson \\ Clinical Research Consortium \\ Singaravelu Jagadeesan \\ Wake Research Associates \\ Evgeny Fink \\ SPRI Clinical Trials \\ Ming-Chyi Pai ( $\nabla$ tsailing-lu@a2healthcare.com ) \\ Division of Behavioral Neurology, Department of Neurology, National Cheng Kung University Hospital, College of \\ Medicine, National Cheng Kung University, Tainan City, Taiwan https://orcid.org/0000-0002-4241-9403
}

\section{Research}

Keywords: BAC, CSTC1, Alzheimer's disease, Vascular dementia, MMSE, Mixed dementia

Posted Date: September 28th, 2020

DOI: https://doi.org/10.21203/rs.3.rs-81331/v1

License: (c) This work is licensed under a Creative Commons Attribution 4.0 International License. Read Full License 


\section{Abstract}

Background: Dementia causes a long-term and gradual cognitive decline and there is no treatment to slow or stop the progression of dementia.

Methods: Middle cerebral artery occlusion in rats was employed to investigate the effects of BAC in neural inflammation. A phase II study was conducted in Alzheimer's disease or vascular dementia patients. BAC or matched vehicle was applied topically on the subject's external nasal skin, scalp, and neck, twice daily, 30 grams per day for 12 weeks. Cognitive evaluation questionnaires including ADAS-Cog, ADCS-ADL, MMSE, NPI, CIBIS, and CIBICplus scores were performed to evaluate the efficacy. A responder analysis was conducted with stratification of different sub-groups of receiving/not receiving dementia medication or donepezil hydrochloride and type of dementia.

Results: The responder analysis showed that the naïve and not using donepezil hydrochloride sub-groups in the BACtreated group had a higher responder rate compared to using donepezil hydrochloride and any dementia medication sub-groups. The BAC group showed a higher percentage of responders than the vehicle group in the mixed dementia population.

Conclusion: BAC was safe for human use. BAC improved the responder rate in naïve dementia patients regardless of dementia type and in patients not receiving donepezil hydrochloride. The bigger sample size is required to confirm the efficacy of BAC.

Trial registration: NCT02886494 (First posted: September 1, 2016)

\section{Background}

Dementia is a progressive syndrome accompanied by the decline of intelligence which causes disability among older people [1]. Alzheimer's disease (AD) is the major cause of dementia, larger than all other causes combined [2,3]. Mixed dementia is the second most frequent cause and is defined as multiple types of dementias occurring simultaneously. Until now, only two classes of drugs are approved by the FDA to treat the cognitive symptoms of AD: cholinesterase inhibitors (donepezil, rivastigmine, and galantamine) and noncompetitive N-methyl D-aspartate (NMDA) antagonist (memantine) [4]. However, cholinesterase inhibitors are related to excessive accumulation of acetylcholine at neuromuscular junctions and synapses, which frequently cause mild-to-moderate nausea and vomiting [5-8]. For patients treated with NMDA antagonist, mild side effects including confusion, dizziness, headache, sedation, agitation, falls, and constipation were reported [9]. In addition, there was no strong evidence to support that patients with vascular dementia $(\mathrm{VaD})$ or mixed dementia would benefit from these drugs $[10,11]$.

Natural products are considered as an alternative treatment for AD in recent years. The neuroprotective properties of soy isoflavone have been examined in several studies $[12,13]$. Soybean isoflavones have been known for the neuroprotective effects, and the possible mechanism is associated with $\beta$ amyloid clearance and anti-vascular inflammation [14]. Genistein, one of the soy isoflavones, has been demonstrated the neuroprotective effects from amyloid $\beta$-induced neurotoxicity through the estrogen receptor-mediated pathway and the elimination of the free radicals $[15,16]$. Although the results are still controversial, there are increasing interests in isoflavones in the protection from neurodegeneration [15-17].

BAC (also called CSTC1, CHARSIRE Trauma Complex 1), a component of soybean extraction developed by Charsire Biotechnology Corp, Taiwan, is under the investigation for the treatments of a variety of diseases including $A D$ and VaD. BAC is a vapor fraction from seeds of Glycine max (L.) Merr. BAC was shown to promote the proliferation of neuroblastoma IMR-32 cells and this observation may correlate to the improvement of the cognitive performance in the 
heavy metal-induced $A D$ mice and the VaD-resembled rats when treated with BAC (unpublished data). In the present study, we investigated the inflammatory molecule expression in the brain tissues of the middle cerebral artery occlusion (MCAO) rats following BAC treatment. Besides, we are presenting here a randomized, double-blind, vehicle-controlled, parallel, phase II study was designed to evaluate the efficacy and safety of BAC in patients with AD, VaD, or mixed dementia. The primary objective of the clinical study was to evaluate the efficacy of BAC in patients with AD or VaD. The secondary objective of this study was to evaluate the safety of BAC patients with AD or VaD.

\section{Materials And Methods}

\section{Middle cerebral artery occlusion (MCAO) rats as an animal model of dementia}

A total of 18 male Wistar rats (8 weeks old) weighing 250-330 grams were purchased from the BioLASCO Taiwan Co., Ltd. Rats were randomly divided into three groups ( $N=6$ in each group): sham group, middle cerebral artery occlusion (MCAO) group, and MCAO + BAC group: treated with BAC for 7 days after MCAO. The procedure of MCAO from previously reported studies was followed and described as below $[18,19]$. Rats were anesthetized by $3 \%$ isoflurane in oxygen and maintained anesthetized using $2 \%$ isoflurane in oxygen. A ventral midline incision was made to identify the left common carotid artery (LCCA), external carotid artery (ECA), and internal carotid artery (ICA). The ECA and the proximal LCCA were tied with a 6 - 0 silk suture, followed by blocking ICA blood flow with an arterial clip. The blood flow blocking procedure was conducted by using proper embolization wire (MCAO sutures, per weight of the rat) to insert from the distal end of LCCA and enter the brain through ICA. After inserted an $18 \mathrm{~mm}$ of nylon suture, the resistance that was felt indicated the blockage of the middle cerebral artery and caused cerebral ischemia. After 45 minutes of ischemia, the embolization wire was removed to allow blood reperfusion. The incision was closed and animals were transferred to the cages. The rats in the MCAO + BAC group were applied with 2 grams of BAC to the whole area of head and neck following by the gentle massage for 30 seconds, and then BAC was applied to the nose for each rat. Topical BAC treatment was repeated for 1 week and the brain tissues were collected for further analysis.

All the experimental procedures were performed according to the specifications of the Guideline for Animal Experiments of Animal Center, Kaohsiung Medical University Hospital, Kaohsiung, Taiwan.

\section{Western blot analysis}

To prepare protein extraction, the frozen brains were homogenized in T-PER ${ }^{\mathrm{TM}}$ reagent (Tissue Protein Extraction Reagent, \#78510, Thermo Fisher Scientific/Invitrogen, Carlsbad, CA) supplemented with a cocktail of protease inhibitors (Complete ${ }^{\mathrm{TM}}$ Protease Inhibitor Cocktail, Roche/Sigma Chemical Co., St. Louis, MO), followed by centrifugation at $10000 \times \mathrm{g}$ for 10 minutes to harvest the supernatant. $50 \mu \mathrm{g}$ total protein for each sample was loaded in $12 \%$ SDS gel. After electrophoresis, the protein was transferred to polyvinylidene difluoride (PVDF) membranes. After blocking for 1 hour with blocking buffer ( $5 \%$ of BSA in TBST), the membranes were probed with primary antibodies overnight at $4{ }^{\circ} \mathrm{C}$. The following antibodies were used: IL-1 $\beta$ (1:500; \#500-P80; Peprotech, New Jersey, USA) and GAPDH (1:50000; \#6004-1-Ig; Proteintech, Chicago, USA). After washing, the membranes were incubated with horseradish peroxidase (HRP) conjugated anti-mouse or anti-rabbit secondary antibody (Jackson ImmunoResearch, PA, USA) at room temperature for 1 hour. The blots were developed using chemiluminescence, captured by CCD chemiluminescence imaging system (ChemiDoc XRS Imaging System, Bio-Rad, Hercules, CA) and the intensity of each band was quantified using NIH Image J software.

\section{Clinical study design and population}


For this clinical study, it was a randomized, double-blind, vehicle-controlled, and parallel phase II trial to evaluate the efficacy and safety of BAC in patients with $A D, V a D$, or mixed dementia. The sample size of treatment and control groups was 45 versus 15 patients (3:1). The study participants were recruited from 10 study centers across the United States from January 30, 2017 to November 01, 2018.

The main inclusion and exclusion criteria were described below and may be referred to NCT02886494 at ClinicalTrials.gov for details. At each study site, eligible patients were stratified to either AD (including mixed dementia) or non-Alzheimer's disease.

The main inclusion criteria were (1) with a diagnosis of one of the following diseases: i. VaD according to the NINDSAIREN International Workshop criteria, ii. AD according to the NIAAA criteria, or iii. mixed dementia (possible AD with the cerebrovascular disease) according to the NIAAA criteria; (2) with mild-to-moderate dementia (score of the MMSE defined as between 10 to 24 and score of ADAS-Cog as at least 12). The main exclusion criteria were (1) with large vessel thrombosis (thrombotic stroke occurring in large arteries); (2) with radiological evidence of other brain disorders (subdural hematoma, post-traumatic/post-surgery); (3) with dementia caused by other brain diseases except for AD and $\mathrm{VaD}$.

\section{Outcomes}

The primary endpoint of this study was the change in Alzheimer's Disease Assessment Scale-Cognitive Subscale (ADAS-Cog) score at Week 12 compared to baseline, which measures the severity of the most prominent symptoms of $A D$ including disturbances in memory, language, praxis, and attention.

Secondary endpoints included changes in ADAS-cog score at other visits; CIBIC-plus score at all visits, which is the Clinician's Interview-Based Impression of Severity/Clinician's Interview-Based Impression of Change Plus Caregiver Input (CIBIS/CIBIC-Plus), a widely used in anti-dementia drug trials for global assessment in the outcome measurement [17]; change in Alzheimer's Disease Cooperative Study-Activities of Daily Living (ADCS-ADL) Inventory compared to baseline, which measures the competence of patients with $A D$ in their basic and instrumental activities of daily living [20]; change in Mini-Mental State Examination (MMSE) at all visits compared to baseline, which evaluates cognitive functions among elderly on orientation, attention, memory, language, and visual-spatial skills [21]; and change in Neuropsychiatric Instrument (NPI) score compared to baseline, which assesses neuropsychiatric symptoms and psychopathology of patients with $A D$ and other neurodegenerative disorders.

The "responder" analyses of the change in ADAS-Cog and MMSE score from the baseline to Week 4, 8, and 12 were performed. The "responder" analyses consisted of two steps: (1) The ADAS-Cog/MMSE score at Week 4, 8, or 12 was deducted by the ADAS-Cog/MMSE score at the baseline; (2) based on the score difference, patients were then dichotomized into "responder" or "non-responder". If a patient made progress or remained unchanged in ADASCog/MMSE score, he/she was considered as a "responder", whereas a patient who deteriorated in ADAS-Cog/MMSE score was considered as a "non-responder". The patients were further stratified into 12 subgroups of receiving/not receiving any dementia medication (DM), receiving/not receiving any donepezil hydrochloride (DH), and type of dementia (all patients, AD patients, and mixed dementia patients).

The safety measurements included the incidence of adverse events and changes in physical examinations and laboratory monitoring parameters.

\section{Statistical analysis}

Changes in the efficacy scores including ADAS-cog score, ADCS-ADL score, MMSE score, and NPI score were performed by ANCOVA with the treatment group as a factor and with baseline score as a covariate. The CIBIS/CIBIC-plus score at 
all post-treatment visits was presented by the transition table. The Fisher's exact test was performed to test the "responder" differences in ADAS-Cog and MMSE scores between treatment groups. The safety outcomes determined by the incidence of adverse events were reported by treatment groups and by physiological systems. All treatment group comparisons were conducted with a significance level of 0.05, using 2-tailed tests. All the statistical analyses were performed by Statistical Analysis Software (SAS) 9.4 (SAS institute September 2017).

\section{Results}

\section{BAC treatment reduced the expression of pro-IL-1 $\beta$ in MCAO rats}

The middle cerebral artery occlusion (MCAO) ischemic stroke model was considered to be a reliable model to study dementia. We evaluated the anti-inflammation effects of BAC in MCAO rats with BAC topical treatment by 7 days after cerebral ischemia reperfusion. The cytokine interleukin-1 $\beta$ (IL-1 $\beta$ ) has been implicated in the contribution to ischemic brain damage [22]. Western blot showed that pro-IL-1 $\beta$ (precursor of IL-1 $\beta$ ) significantly increased in the MCAO group compared to the sham group (Fig. 1). This confirmed a successful induction of ischemic stroke in the rats. Treatment with BAC significantly decreased the pro-IL-1 $\beta$ expression in the brain of MCAO + BAC rats (Fig. 1). This result indicated that BAC treatment may reduce brain inflammation in MCAO rats.

\section{Demographics, dementia history, and drug exposure}

To further explore the efficacy and safety of BAC in patients with $A D, V a D$ or mixed dementia, a clinical trial was conducted in the US. Details of subject disposition are shown in Fig. 2. In total 119 patients were screened, and 80 eligible study patients were enrolled in the study, with sixty patients being randomized to BAC treated group and twenty patients to vehicle-treated group. A total of 21 patients did not complete the study, and among them, 15 patients voluntarily withdrew consent.

Generally, the patients in the BAC and vehicle groups showed similar baseline characteristics, including age, gender, body weight, height, body mass index (BMI), and race. The mean duration of dementia history was 3.64 years. A total of $55(68.8 \%), 17(21.3 \%)$, and 8 (10.0\%) patients were with Alzheimer's disease (AD), mixed dementia, and vascular dementia $(\mathrm{VaD})$, respectively. There was no particular group difference regarding dementia types and duration. The demographic characteristics, dementia history, and the extent of drug exposure are summarized in Table 1. The duration of exposure to BAC versus vehicle was 76.7 days versus 73.9 days. No statistically significant difference in the extent of drug exposure was observed between treatment groups. The details of drug exposure are also summarized in Table 1. 
Table 1

The demographics, dementia history, and extent of drug exposure

\begin{tabular}{|c|c|c|c|c|}
\hline & $\begin{array}{l}\mathrm{BAC} \\
\mathrm{N}=60\end{array}$ & $\begin{array}{l}\text { Vehicle } \\
N=20\end{array}$ & $\begin{array}{l}\text { Total } \\
\mathbf{N}=\mathbf{8 0}\end{array}$ & $P$ value \\
\hline \multicolumn{5}{|l|}{ Demographics } \\
\hline Age (years) ${ }^{1}$ & $73.2 \pm 9.6$ & $73.1 \pm 9.2$ & $73.2 \pm 9.5$ & $0.973^{2}$ \\
\hline Female $(\mathrm{N}, \%)$ & $41(68.3 \%)$ & $11(55.0 \%)$ & $52(65.0 \%)$ & $0.279^{3}$ \\
\hline \multicolumn{5}{|l|}{ Race (N, \%) } \\
\hline Caucasian & $54(90.0 \%)$ & $17(85.0 \%)$ & $71(88.8 \%)$ & \multirow[t]{4}{*}{$0.478^{4}$} \\
\hline African American & $5(8.3 \%)$ & $2(10.0 \%)$ & $7(8.8 \%)$ & \\
\hline Hispanic & $1(1.7 \%)$ & $0(0.0 \%)$ & $1(1.3 \%)$ & \\
\hline Native American & $0(0.0 \%)$ & $1(5.0 \%)$ & $1(1.3 \%)$ & \\
\hline Body Weight $(\mathrm{kg})^{1}$ & $79.2 \pm 21.5$ & $78.0 \pm 10.5$ & $78.9 \pm 19.3$ & $0.674^{5}$ \\
\hline Body Height $(\mathrm{cm})^{1}$ & $162.6 \pm 9.9$ & $167.8 \pm 11.4$ & $163.9 \pm 10.5$ & $0.065^{5}$ \\
\hline $\mathrm{BMI}\left(\mathrm{kg} / \mathrm{m}^{2}\right)^{1}$ & $29.9 \pm 7.4$ & $27.8 \pm 3.5$ & $29.4 \pm 6.7$ & $0.413^{5}$ \\
\hline \multicolumn{5}{|l|}{ Dementia Type (N, \%) } \\
\hline Alzheimer's Disease & $42(70.0 \%)$ & $13(65.0 \%)$ & $55(68.8 \%)$ & \multirow[t]{3}{*}{$0.424^{4}$} \\
\hline Mixed Dementia & $11(18.3 \%)$ & $6(30.0 \%)$ & $17(21.3 \%)$ & \\
\hline Vascular Dementia & $7(11.7 \%)$ & $1(5.0 \%)$ & $8(10.0 \%)$ & \\
\hline Dementia Duration (years) ${ }^{1}$ & $3.9 \pm 3.0$ & $2.9 \pm 2.6$ & $3.6 \pm 2.9$ & $0.111^{5}$ \\
\hline \multicolumn{5}{|l|}{ Extent of Drug Exposure ${ }^{1}$} \\
\hline Drug Exposure Duration (Days) & $76.7 \pm 30.1$ & $73.9 \pm 23.1$ & $69.2 \pm 28.6$ & $0.872^{5}$ \\
\hline Drug Application Count (Times) & $131.3 \pm 60.2$ & $144.4 \pm 46.4$ & $134.4 \pm 57.2$ & $0.877^{5}$ \\
\hline Application Frequency (Times/day) & $1.9 \pm 0.2$ & $1.9 \pm 0.1$ & $1.9 \pm 0.2$ & $0.395^{5}$ \\
\hline
\end{tabular}

\section{Efficacy outcomes and estimations}

\section{Primary Endpoint}

The primary efficacy outcome was the change in the ADAS-Cog score at Week 12 compared to baseline. After 12 weeks of BAC treatment, patients showed improvement in ADAS-Cog score by 0.7 , which was not significantly different from patients treated with vehicle (Table 2). 
Table 2

The efficacy analysis

\begin{tabular}{|c|c|c|c|c|c|c|c|c|c|}
\hline \multirow[b]{2}{*}{ BAC } & \multicolumn{3}{|c|}{ Week 4 - Baseline } & \multicolumn{3}{|c|}{ Week 8 - Baseline } & \multicolumn{3}{|c|}{ Week 12 - Baseline } \\
\hline & Vehicle & $P$ value & BAC & Vehicle & $P$ & BAC & Vehicle & $P$ value & \\
\hline 51 & 18 & & 48 & 17 & value & 57 & 19 & & \\
\hline \multirow[t]{2}{*}{ ADAS-Cog ${ }^{1}$} & -0.5 & 2.3 & $0.116^{3}$ & -0.8 & -2.1 & $0.314^{3}$ & -0.7 & -1.6 & $0.568^{3}$ \\
\hline & \pm 4.1 & \pm 4.4 & & \pm 4.2 & \pm 5.1 & & \pm 6.0 & \pm 4.7 & \\
\hline \multirow[t]{2}{*}{$\mathrm{MMSE}^{1}$} & 0.6 & 0.2 & $0.463^{3}$ & 0.3 & 1.4 & $0.057^{3}$ & 0.5 & 0.6 & $0.625^{2}$ \\
\hline & \pm 2.1 & \pm 2.4 & & \pm 1.7 & \pm 2.7 & & \pm 2.6 & \pm 2.3 & \\
\hline \multirow[t]{2}{*}{ ADCS-ADL ${ }^{1}$} & -0.7 & 1.2 & $0.084^{3}$ & 0.1 & 0.5 & $0.703^{3}$ & -0.4 & 1.3 & $0.186^{2}$ \\
\hline & \pm 3.6 & \pm 4.6 & & \pm 3.2 & \pm 6.1 & & \pm 4.5 & \pm 5.6 & \\
\hline \multirow[t]{2}{*}{ NPI-10 FxS ${ }^{1}$} & -0.4 & -2.3 & $0.501^{2}$ & -0.8 & -2.8 & $0.345^{2}$ & -1.3 & -2.3 & $0.534^{2}$ \\
\hline & \pm 8.6 & \pm 7.0 & & \pm 4.0 & \pm 6.3 & & \pm 6.7 & \pm 6.7 & \\
\hline \multirow[t]{2}{*}{$\mathrm{NPI}-10 \mathrm{CDS}^{1}$} & -0.0 & -1.9 & $0.328^{2}$ & -0.5 & -2.1 & $0.394^{2}$ & -0.7 & -2.5 & $0.722^{2}$ \\
\hline & \pm 2.8 & \pm 4.1 & & \pm 1.9 & \pm 4.5 & & \pm 2.6 & \pm 4.5 & \\
\hline \multirow[t]{2}{*}{$\mathrm{NPI}-12 \mathrm{FxS}^{1}$} & -0.6 & -3.9 & $0.474^{2}$ & -1.3 & -4.8 & $0.305^{2}$ & -2.1 & -4.1 & $0.443^{2}$ \\
\hline & \pm 9.9 & \pm 9.1 & & \pm 6.4 & \pm 9.4 & & \pm 9.9 & \pm 10.2 & \\
\hline \multirow[t]{2}{*}{$\mathrm{NPI}-12 \mathrm{CDS}^{1}$} & -0.0 & -2.6 & $0.204^{2}$ & -0.7 & -2.8 & $0.157^{2}$ & -0.9 & -3.2 & $0.861^{2}$ \\
\hline & \pm 3.0 & \pm 5.2 & & \pm 2.3 & \pm 5.8 & & \pm 3.3 & \pm 5.8 & \\
\hline \multicolumn{10}{|c|}{$\begin{array}{l}\text { 1. ADAS-Cog, Alzheimer's Disease Assessment Scale- Cognitive; MMSE, Mini-Mental State Examination; ADCS-ADL, } \\
\text { Alzheimer's Disease Cooperative Study Activities of Daily Living; NPI-10 FXS, Neuropsychiatric Inventory } 10 \text { (10- } \\
\text { item) Frequency X Severity; NPI-10 CDS, Neuropsychiatric Inventory } 10 \text { (10-item) Caregiver Distress Score; NPI- } \\
12 \text { FXS, Neuropsychiatric Inventory } 12 \text { (12-item) Frequency X Severity; NPI-12 CDS, Neuropsychiatric Inventory } 12 \\
\text { (12-item) Caregiver Distress Score; Values are given as Mean } \pm \text { SD. 2. P value was calculated by ANCOVA, 3. P value } \\
\text { was calculated by ANOVA. }\end{array}$} \\
\hline
\end{tabular}

\section{Secondary Endpoints}

The secondary efficacy endpoints included changes from baseline in ADAS-Cog score at Week 4 and Week 8 visits, and changes from baseline to Week 4, 8, and 12 in MMSE, ADCS-ADL, NPI, and CIBIC/CIBIC-plus scores.

Patients treated with BAC showed a mean of $0.5 \pm 4.1$ worsening and $0.8 \pm 4.2$ improvement ADAS-Cog score at Week 4 and Week 8, respectively, which was not significantly different from vehicle-treated patients. In the MMSE test, patients treated with BAC group showed slight improvements of $0.6 \pm 2.1,0.3 \pm 1.7$, and $0.5 \pm 2.6$ at Week 4, 8, and 12, respectively, which was also not significantly different from vehicle-treated patients, that showed fluctuation of improvement of $0.2 \pm 2.4,1.4 \pm 2.7$, and $0.6 \pm 2.3$ at Week 4,8 , and 12 respectively. When being assessed by the ADCSADL score, patients treated with BAC did not show improvement at the end of the treatment period, but those treated with vehicle showed a slight improvement in ADCS-ADL. The NPI questionnaires were behavior measurement tools used in this study, and the information from NPI was obtained from the caregiver of that patient. Both 10-item and 12item versions of NPI were used, measuring frequency, severity, and distress level of patient behavior. Patients treated with BAC generally showed no improvement in behavior by Week 4, slight improvements in behavior by Week 8 , and 12 . In contrast, patients treated with vehicle appeared to show score reduction as early as Week 4, but the scores fluctuated 
in the remaining period of the study. The results of secondary efficacy endpoints were summarized in Table 2. As for CIBIS/CIBIC-plus analysis, the distribution of CIBIS/CIBIC-plus score was similar in the BAC and vehicle-treated groups throughout the study period (Table 3).

Table 3

The CIBIS/CIBIC-plus score

\begin{tabular}{|c|c|c|c|c|}
\hline CIBIS/CIBIC-plus & BAC & Vehicle & Total & p-value ${ }^{1}$ \\
\hline Baseline & 60 & 20 & 80 & 0.2638 \\
\hline $\mathrm{N}$ & $34(56.7 \%)$ & $14(70.0 \%)$ & $48(60.0 \%)$ & \\
\hline 3- Mildly ill & $24(40.0 \%)$ & $6(30.0 \%)$ & $30(37.5 \%)$ & \\
\hline 4- Moderately ill & $2(3.3 \%)$ & $0(0.0 \%)$ & $2(2.5 \%)$ & \\
\hline \multicolumn{5}{|l|}{ 5- Markedly ill } \\
\hline Week 4 & 51 & 18 & 69 & 0.6382 \\
\hline $\mathrm{N}$ & $1(2.0 \%)$ & $1(5.6 \%)$ & $2(2.9 \%)$ & \\
\hline 2- Much improved & $6(11.8 \%)$ & $3(16.7 \%)$ & $9(13.0 \%)$ & \\
\hline 3- Minimal improved & $41(80.4 \%)$ & $10(55.6 \%)$ & $51(73.9 \%)$ & \\
\hline 4- No change & $3(5.9 \%)$ & $4(22.2 \%)$ & $7(10.1 \%)$ & \\
\hline \multicolumn{5}{|l|}{ 5- Minimal worsening } \\
\hline Week 8 & 48 & 17 & 65 & 0.3570 \\
\hline $\mathrm{N}$ & $1(2.1 \%)$ & $1(5.9 \%)$ & $2(3.1 \%)$ & \\
\hline 2- Much improved & $2(4.2 \%)$ & $1(5.9 \%)$ & $3(4.6 \%)$ & \\
\hline 3- Minimal improved & $36(75.0 \%)$ & $13(76.5 \%)$ & $49(75.4 \%)$ & \\
\hline 4- No change & $8(16.7 \%)$ & $2(11.8 \%)$ & $10(15.4 \%)$ & \\
\hline 5- Minimal worsening & $1(2.1 \%)$ & $0(0.0 \%)$ & $1(1.5 \%)$ & \\
\hline \multicolumn{5}{|l|}{ 6- Moderate worsening } \\
\hline Week 12 & 44 & 16 & 60 & 0.9143 \\
\hline $\mathrm{N}$ & $1(2.3 \%)$ & $0(0.0 \%)$ & $1(1.7 \%)$ & \\
\hline 1- Very much improved & $1(2.3 \%)$ & $2(12.5 \%)$ & $3(5.0 \%)$ & \\
\hline 2- Much improved & $2(4.5 \%)$ & $0(0.0 \%)$ & $2(3.3 \%)$ & \\
\hline 3- Minimal improved & $33(75.0 \%)$ & $11(68.8 \%)$ & $44(73.3 \%)$ & \\
\hline 4- No change & $5(11.4 \%)$ & $3(18.8 \%)$ & $8(13.3 \%)$ & \\
\hline 5- Minimal worsening & $2(4.5 \%)$ & $0(0.0 \%)$ & $2(3.3 \%)$ & \\
\hline \multicolumn{5}{|l|}{ 6- Moderate worsening } \\
\hline 1. Mantel-Haensze & & & & \\
\hline
\end{tabular}




\section{MMSE responder analysis for all, $A D$, and mixed dementia patients}

A responder analysis was carried out exploring the MMSE response at Week 4, 8, and 12 in different subgroups, and the subgroups included patients who are naïve to dementia medication (naïve, group a, and e), patients who are currently taking dementia medication (DM use, group $b$, and f), patients who are not receiving donepezil hydrochloride (No $\mathrm{DH}$, group c, and g), and patients who are receiving donepezil hydrochloride (DH use, group d, and h) (Table 4). Naïve patients and patients who do not take donepezil receiving BAC treatment (group a and c) tend to have consistently more responders in MMSE scores throughout the study when compared to other subgroups receiving either BAC or vehicle. Patients taking donepezil receiving vehicle (group $h$ ) tend to have fewer responders when compared to all the other subgroups receiving vehicle (group e-g). Patients who take donepezil receiving BAC treatment (group d) also tend to have fewer responders compared to all other subgroups receiving BAC (group a-c). When patients were further stratified to $A D$ and mixed dementia patients, similar results of MMSE response were observed (Table 4). Lastly, the results of responders in patients with mixed dementia showed that the patients receiving BAC treatment had more responders than patients treated with the vehicle throughout the study (Table 5).

The responder changes from the baseline to Week 4, 8, or 12 in the MMSE score by sub-groups were demonstrated in Fig. 3. In both BAC and vehicle groups, patients who are naïve to dementia medication and patients who do not use donepezil have greater responders throughout the study and the percentage of responders tended to increase by time. In contrast, the patients who use dementia medication and patients who use donepezil receiving BAC treatment gradual decline in responders in time, and those receiving vehicle treatment showed fluctuation in the percentage of responders. 
The responder analysis of MMSE score for all, AD and mixed dementia patients

\begin{tabular}{lllllllll}
$\begin{array}{l}\text { MMSE responder } \\
\text { analysis }\end{array}$ & BAC & \multicolumn{9}{c}{ Vehicle } \\
& Naïve & DM Use & No & DH Use & Naïve & DM Use & No & DH \\
& (a) & (b) & DH & (d) & (e) & (f) & DH & Use \\
& & & (c) & & & (h)
\end{tabular}

\section{All Patients (includes AD, VaD, Mixed dementia)}

\begin{tabular}{|c|c|c|c|c|c|c|c|c|c|}
\hline Week 4 & $\mathrm{~N}$ & 17 & 34 & 29 & 22 & 8 & 10 & 12 & 6 \\
\hline - Baseline & $\begin{array}{l}\text { Responder } \\
(\%)\end{array}$ & $\begin{array}{l}13 \\
(77)\end{array}$ & $\begin{array}{l}26 \\
(77)\end{array}$ & $23(79)$ & $16(73)$ & $5(63)$ & $4(40)$ & $7(58)$ & $2(33)$ \\
\hline Week 8 & $\mathrm{~N}$ & 15 & 33 & 26 & 22 & 8 & 9 & 12 & 5 \\
\hline - Baseline & $\begin{array}{l}\text { Responder } \\
(\%)\end{array}$ & $\begin{array}{l}12 \\
(80)\end{array}$ & $\begin{array}{l}21 \\
(64)\end{array}$ & $21(81)$ & $12(55)$ & $6(75)$ & $7(78)$ & $9(75)$ & $4(80)$ \\
\hline Week 12 & $\mathrm{~N}$ & 21 & 36 & 33 & 24 & 8 & 11 & 13 & 6 \\
\hline Baseline & $\begin{array}{l}\text { Responder } \\
\text { (\%) }\end{array}$ & $\begin{array}{l}17 \\
(81)\end{array}$ & $\begin{array}{l}22 \\
(61)\end{array}$ & 27 (82) & $12(50)$ & $6(75)$ & $6(55)$ & $9(69)$ & $3(50)$ \\
\hline
\end{tabular}

\section{Alzheimer's Disease (AD) Patients}

\begin{tabular}{|c|c|c|c|c|c|c|c|c|c|}
\hline Week 4 & $\mathrm{~N}$ & 9 & 27 & 17 & 19 & 5 & 7 & 6 & 6 \\
\hline Baseline & $\begin{array}{l}\text { Responder } \\
\text { (\%) }\end{array}$ & $\begin{array}{l}6 \\
(67)\end{array}$ & $\begin{array}{l}20 \\
(74)\end{array}$ & $12(71)$ & $14(74)$ & $3(60)$ & $3(43)$ & $4(67)$ & 2 (33) \\
\hline Week 8 & $\mathrm{~N}$ & 9 & 26 & 16 & 19 & 5 & 6 & 6 & 5 \\
\hline Baseline & $\begin{array}{l}\text { Responder } \\
(\%)\end{array}$ & $\begin{array}{l}7 \\
(78)\end{array}$ & $\begin{array}{l}15 \\
(58)\end{array}$ & $12(75)$ & $10(53)$ & $4(80)$ & $5(83)$ & $5(83)$ & $4(80)$ \\
\hline Week 12 & $\mathrm{~N}$ & 11 & 29 & 19 & 21 & 5 & 8 & 7 & 6 \\
\hline Baseline & $\begin{array}{l}\text { Responder } \\
\text { (\%) }\end{array}$ & $\begin{array}{l}9 \\
(82)\end{array}$ & $\begin{array}{l}17 \\
(59)\end{array}$ & 15 (79) & $11(52)$ & $3(60)$ & $4(50)$ & $4(57)$ & $3(50)$ \\
\hline \multicolumn{10}{|c|}{ Mixed Dementia Patients } \\
\hline Week 4 & $\mathrm{~N}$ & 5 & 5 & 7 & 3 & 2 & 3 & 5 & $\mathrm{~N} / \mathrm{A}$ \\
\hline - Baseline & $\begin{array}{l}\text { Responder } \\
\text { (\%) }\end{array}$ & $\begin{array}{l}5 \\
(100)\end{array}$ & $\begin{array}{l}4 \\
(80)\end{array}$ & 7 (100) & $2(67)$ & $2(100)$ & 1 (33) & $3(60)$ & \\
\hline Week 8 & $\mathrm{~N}$ & 4 & 5 & 6 & 3 & 2 & 3 & 5 & N/A \\
\hline Baseline & $\begin{array}{l}\text { Responder } \\
(\%)\end{array}$ & $\begin{array}{l}4 \\
(100)\end{array}$ & $\begin{array}{l}4 \\
(80)\end{array}$ & $6(100)$ & $2(67)$ & $2(100)$ & $2(67)$ & $4(80)$ & \\
\hline Week 12 & $\mathrm{~N}$ & 5 & 5 & 7 & 3 & 2 & 3 & 5 & $\mathrm{~N} / \mathrm{A}$ \\
\hline Baseline & $\begin{array}{l}\text { Responder } \\
(\%)\end{array}$ & $\begin{array}{l}5 \\
(100)\end{array}$ & $\begin{array}{l}3 \\
(60)\end{array}$ & 7 (100) & $1(33)$ & $2(100)$ & $2(67)$ & $4(80)$ & \\
\hline
\end{tabular}




\begin{tabular}{|c|c|c|c|c|c|c|c|c|}
\hline \multirow{4}{*}{$\begin{array}{l}\text { MMSE responder } \\
\text { analysis }\end{array}$} & \multicolumn{4}{|l|}{ BAC } & \multicolumn{4}{|c|}{ Vehicle } \\
\hline & Naïve & DM Use & No & DHUse & Naïve & DM Use & No & $\mathrm{DH}$ \\
\hline & (a) & (b) & DH & (d) & (e) & (f) & DH & (h) \\
\hline & & & (c) & & & & (g) & \\
\hline \multicolumn{9}{|c|}{ (a) (e): patients are naïve to any dementia medication (Naïve) } \\
\hline \multicolumn{9}{|c|}{ (b) (f): patients are currently taking any dementia medication (DM) } \\
\hline \multicolumn{9}{|c|}{ (c) (g): patients are not receiving donepezil hydrochloride (No DH) } \\
\hline \multicolumn{9}{|c|}{ (d) (h): patients are receiving donepezil hydrochloride (DH use) } \\
\hline
\end{tabular}

Table 5

The responder analysis of MMSE score for mixed dementia patients

\begin{tabular}{|llll|}
\hline & & BAC & Vehicle \\
\hline Week 4 - Baseline & $\mathrm{N}$ & 10 & 5 \\
& Responder (\%) & $9(90.0)$ & $3(60.0)$ \\
Week 8 - Baseline & $\mathrm{N}$ & 9 & 5 \\
& Responder (\%) & $8(88.9)$ & $4(80.0)$ \\
\hline Week 12 - Baseline & $\mathrm{N}$ & 10 & 5 \\
& Responder (\%) & $8(80.0)$ & $4(80.0)$ \\
\hline
\end{tabular}

\section{ADAS-Cog responder analysis for all, AD, and mixed dementia patients}

A responder analysis was also carried out for ADAS-Cog scores, and the results are shown in Table 6. Naïve patients and patients who have no donepezil use receiving BAC treatment showed more responders throughout the study, compared to other subgroups, and the percentage of responders was found to be maintained at approximately $70 \%$ in all study visits. Similar results were observed when patients were further stratified into patients with AD and patients with mixed dementia. The responder changes from baseline to week 4,8 , or 12 in ADAS-Cog score by sub-groups was illustrated in Fig. 4. In general, in both BAC and vehicle groups, patients who are naïve to dementia medication and patients who do not use donepezil have more responders throughout the study, and those receiving BAC treatment showed an upward slope in the percentage of responders along with the quad-weekly visits. However, for those patients receiving the vehicle, the percentage of responders showed a reduction with a downward trend over time. In addition, patients who are taking dementia medication and patients who are taking donepezil receiving vehicle treatment, fluctuation in responders was observed along with the visits. 
Table 6

The responder analysis of ADAS-Cog score for all, $A D$ and mixed dementia patients

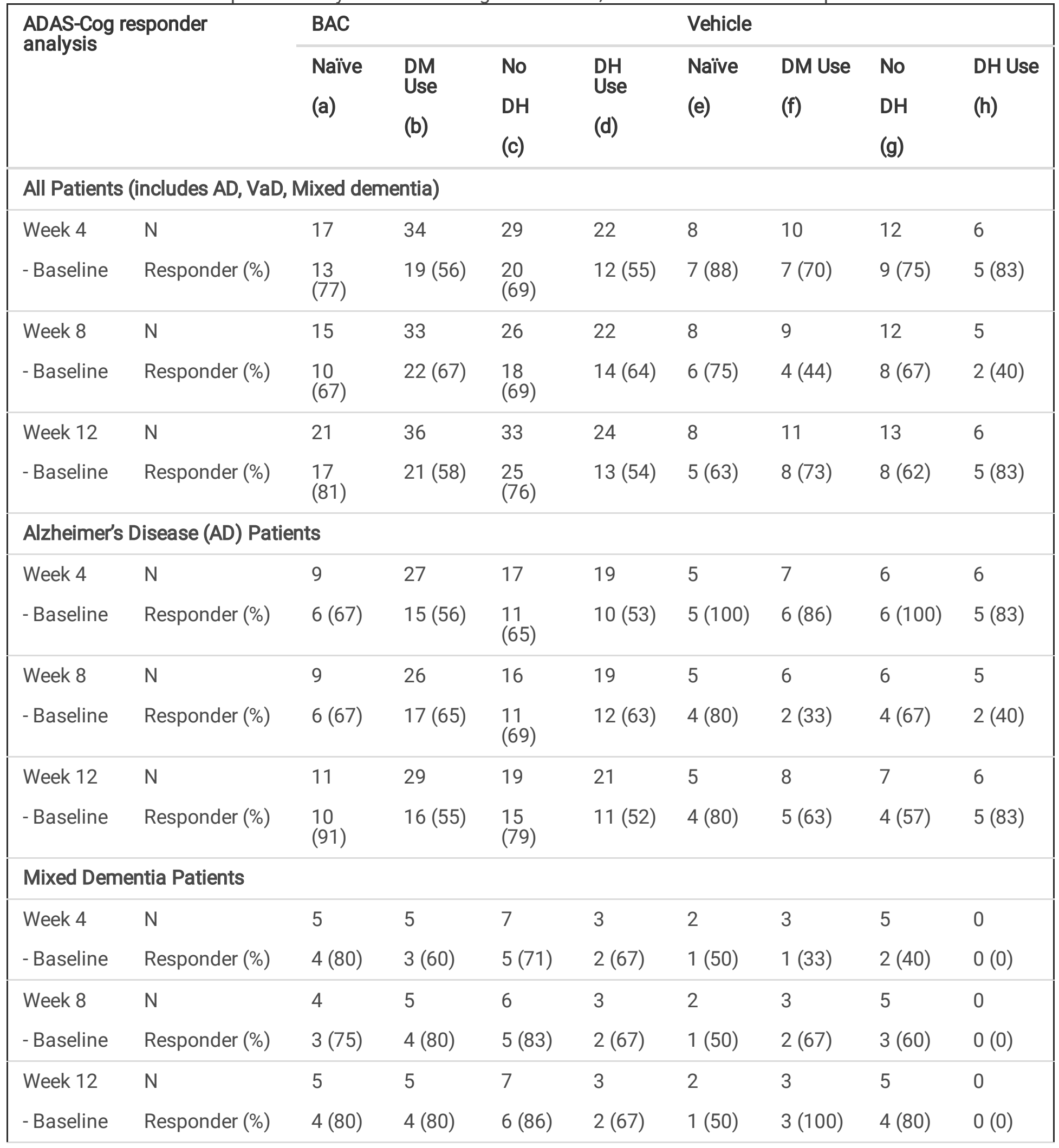




\begin{tabular}{|llllllllll|}
\hline $\begin{array}{l}\text { ADAS-Cog responder } \\
\text { analysis }\end{array}$ & \multicolumn{1}{l}{ BAC } & \multicolumn{9}{c}{ Vehicle } & & \\
\cline { 2 - 8 } & Naïve & DM & No & DH & Naïve & DM Use & No & DH Use \\
& (a) & Use & (b) & DH & Use & (d) & (f) & DH & (h) \\
& & (b) & (c) & (d) & & & (g) &
\end{tabular}

(a) (e): patients are naïve to any dementia medication (Naïve)

(b) (f): patients are currently taking any dementia medication (DM)

(c) (g): patients are not receiving donepezil hydrochloride (No DH)

(d) (h): patients are receiving donepezil hydrochloride (DH use)

\section{Safety results}

There were no serious adverse events or death reported in the trial. In total, 20 (33.3\%) BAC and 7 (35.0\%) vehicletreated patients, experienced at least 1 adverse event $(A E)$. The most prevalent System Organ Classes of AE were "skin and subcutaneous tissue disorders" (11.7\% in BAC, $10.0 \%$ in vehicle, and $11.3 \%$ in total). All other System Organ Classes were below $10 \%$ in the prevalence of the total population. For the treatment-related $A E$, there were 10 (16.7\%) BAC and $2(10.0 \%)$ vehicle-treated patients who experienced at least $1 \mathrm{AE}$ related to the administration of study medication. The most frequent AEs were of "skin and subcutaneous tissue disorders" and was experienced by 5 ( $8.3 \%$ ) BAC and 1 (5.0\%) vehicle-treated patients. The treatment-related AEs are summarized in Table 7. 
Table 7

The incidence of treatment-related adverse event by System Organ Class

\begin{tabular}{|c|c|c|c|}
\hline System Organ Class / Preferred Term & BAC & Vehicle & Total \\
\hline Subject $(\mathrm{N})$ & 60 & 20 & 80 \\
\hline At least one below, $\mathrm{N}(\%)$ & $10(16.7 \%)$ & $2(10.0 \%)$ & $12(15.0 \%)$ \\
\hline Ear disorders & $1(1.7 \%)$ & $0(0.0 \%)$ & $1(1.3 \%)$ \\
\hline Eye irritation & $1(1.7 \%)$ & $0(0.0 \%)$ & $1(1.3 \%)$ \\
\hline General disorders and administration site conditions & $1(1.7 \%)$ & $0(0.0 \%)$ & $1(1.3 \%)$ \\
\hline Application site pain & $1(1.7 \%)$ & $0(0.0 \%)$ & $1(1.3 \%)$ \\
\hline Musculoskeletal and connective tissue disorders & $1(1.7 \%)$ & $0(0.0 \%)$ & $1(1.3 \%)$ \\
\hline Arthralgia & $1(1.7 \%)$ & $0(0.0 \%)$ & $1(1.3 \%)$ \\
\hline Nervous system disorders & $3(5.0 \%)$ & $1(5.0 \%)$ & $4(5.0 \%)$ \\
\hline Dizziness & $0(0.0 \%)$ & $1(5.0 \%)$ & $1(1.3 \%)$ \\
\hline Headache & $3(5.0 \%)$ & $0(0.0 \%)$ & $3(3.8 \%)$ \\
\hline Somnolence & $0(0.0 \%)$ & $1(5.0 \%)$ & $1(1.3 \%)$ \\
\hline Psychiatric disorders & $2(3.3 \%)$ & $0(0.0 \%)$ & $2(2.5 \%)$ \\
\hline Impulsive behavior & $1(1.7 \%)$ & $0(0.0 \%)$ & $1(1.3 \%)$ \\
\hline Nightmare & $1(1.7 \%)$ & $0(0.0 \%)$ & $1(1.3 \%)$ \\
\hline Skin and subcutaneous tissue disorders & $5(8.3 \%)$ & $1(5.0 \%)$ & $6(7.5 \%)$ \\
\hline Dry skin & $1(1.7 \%)$ & $0(0.0 \%)$ & $1(1.3 \%)$ \\
\hline Hair growth abnormal & $0(0.0 \%)$ & $1(5.0 \%)$ & $1(1.3 \%)$ \\
\hline Pain of skin & $1(1.7 \%)$ & $0(0.0 \%)$ & $1(1.3 \%)$ \\
\hline Pruritus & $1(1.7 \%)$ & $0(0.0 \%)$ & $1(1.3 \%)$ \\
\hline Rash & $2(3.3 \%)$ & $0(0.0 \%)$ & $2(2.5 \%)$ \\
\hline
\end{tabular}

\section{Discussion}

$A D$ is the common cause of dementia worldwide, and it is a health issue facing economic costs especially due to the aging population in recent years. As FDA approved drugs are limited, with some reserved for greater severity of dementia, new therapeutics that are safe are in great demand. Our study aimed to explore the possible mechanisms of BAC and understand the efficacy and safety of BAC in patients with Alzheimer's disease or with vascular dementia.

It is widely accepted that brain inflammation has a great impact on the development of dementia. Particularly, IL-1 expression has been found to increase in the AD patients and is associated with $\beta$ amyloid progression [22]. In the MCAO induced ischemic stroke model, the inflammatory markers were analyzed to understand the anti-inflammatory effect of BAC. Our results suggested that BAC can reduce brain inflammation through the route of topical application. Since IL-1 $\beta$ was the only inflammatory marker detected in the present study, further investigation of other inflammatory markers could be conducted. 
In the efficacy assessment, we find no deteriorating effects in cognition, ADL, or behavior in those being treated with BAC. Furthermore, we did find efficacy in specific populations, including patients who are naïve to dementia medications, and patients who are not using donepezil. We suspect that the use of concomitant dementia medications may have confounded the results, and careful patient selection would be essential in further studies.

Donepezil works by altering either the level of acetylcholine or the function of the nicotinic receptor, and the effect of missing a dose will have a rapid effect on the patient's cognition. As new proteins are being constantly synthesized, the level of acetylcholine may fluctuate greatly when patients are not taking these drugs. However, BAC was found to reduce inflammation and maybe not easily affected by a single miss dose of BAC. This can make BAC an attractive therapeutic as it can help patients maintain sustained cognition.

We also discovered that in the mixed dementia population, patients treated with BAC had a greater percentage of responders than those treated with vehicle. Even though with small sample size, BAC may be a beneficial new option for these patients. As there are no medications approved for mixed dementia, these patients are typically prescribed $A D$ medications in an off-label manner. BAC has shown safety and appears as a potential option for these patients.

The safety results indicated that BAC was safe for human use as there was no drug-related death, no drug-related serious adverse events, and no clinically significant changes in laboratory test results, physical examination, and vital signs. In addition, BAC was found to be well-tolerated as only 10 (16.7\%) BAC treated patients experienced at least $1 \mathrm{AE}$ causally related to the administration of study medication. Skin and subcutaneous tissue disorders were the most common class of treatment-related $\mathrm{AE}$, and there were $5 \mathrm{BAC}(8.3 \%)$ and 1 (5.0\%) vehicle-treated patients who experienced skin and subcutaneous tissue disorders.

\section{Limitations}

The present study has some intrinsic limitations to the early stage of clinical development. The sample size of the study was small so it was challenging to find a significant treatment effect on clinical endpoints. Besides, the current study includes data with a short period, meaning that it may be not enough to examine the association between BAC and disease progression. The longer study period will be valuable in future studies.

\section{Conclusions}

In the MCAO induced ischemic model, the topical application of BAC showed an anti-inflammation response. This BAC clinical trial shows that topical BAC application has no deteriorating effects in cognitive performance and may be beneficial to naïve dementia patients as evaluated in the responder analysis. BAC has been found to have a good safety profile in patients with $A D$ or $\mathrm{VaD}$.

\section{Abbreviations}

\section{$A D$}

Alzheimer's disease

\section{ADAS-cog}

Alzheimer Disease Assessment Scale-cognitive

ADCS-ADL

Cooperative Study - Activities of Daily Living

$\mathrm{AE}$

Adverse event

Page 15/22 
CSTC1 (BAC)

CHARSIRE Trauma Complex 1

CIBIC-Plus

Clinician's Interview-Based Impression of Change Plus Caregiver Input CIBIS:Clinician's Interview-Based Impression of Severity

DH

donepezil hydrochloride

DM

dementia medication

ECA

External carotid artery

ICA

Internal carotid artery

IRB

Independent Ethics Committee

IL-1ß

Interleukin 1 beta

LCCA

Left common carotid artery

MCAO

Middle Cerebral Artery Occlusion

MMSE

Mini-Mental State Examination

NIAAA

National Institute on Aging-Alzheimer's Association

NMDA

$\mathrm{N}$-methyl D-asparate

NPI

Neuropsychiatric Instrument

$\mathrm{VaD}$

vascular dementia

\section{Declarations}

\section{Ethics approval and consent to participate}

The study was conducted in accordance with regional regulations and the Declaration of Helsinki. Written informed consent was received from all participants, and ethical approval for data acquisition was provided by Chesapeake IRB (Protocol approval with modification (approval date: 10 October, 2016), approval notice (MOD00206802), continuing review approval (CR00076215) and subject material approval (MOD00203871)). Informed consent was obtained from all study participants and their caregivers before their enrollment.

\section{Consent for publication}

Not applicable.

\section{Availability of data and materials}


The datasets used and/or analyzed during the current study are available from the corresponding authors on reasonable request.

\section{Competing interests}

The authors declare that they have no conflict of interests.

\section{Funding}

Charsire Biotechnology Corporation initiated and sponsored the clinical trial. Financial support for the conduction of the trial and manuscript submission was obtained from the Charsire Biotechnology Corporation.

\section{Author's contributions}

ST, GK, DML, FH, JV, MP, SS, CGA, SJ, and EF conducted and supervised the clinical trial at their affiliated research center. ST also oversaw the progress of trial for all 10 research centers and was a major contributor in writing the manuscript. GK, EF, and MP were contributors in editing and reviewing the manuscript. All authors read and approved the final manuscript.

\section{Acknowledgments}

Our appreciation goes out to all study participants. We also thank the staff at the Charsire Biotechnology Corporation for their secretarial assistance.

\section{Author information}

${ }^{1}$ Pacific Research Network, San Diego, CA 92103, United States

2 Woodland International Research Group, Little Rock, AR 72211, United States

${ }^{3}$ NeuroTrials Research, Inc., Atlanta, GA 30342, United States

${ }^{4}$ Woodland Research Northwest, Rogers, AR 72758, United States

${ }^{5}$ Neurology Diagnostics, Inc., Dayton, $\mathrm{OH} 45414$, United States

6 The Cognitive and Research Center of NJ, Springfield Township, NJ 07081, United States

${ }^{7}$ Advanced Memory Research Institute of NJ, Toms River, NJ 08755, United States

${ }^{8}$ Clinical Research Consortium, Tempe, AZ 85283, United States

${ }^{9}$ Wake Research Associates, Raleigh, NC 27612, United States

10 SPRI Clinical Trials Brooklyn, Brooklyn, NY 11235, United States

${ }^{11}$ Division of Behavioral Neurology, Department of Neurology, National Cheng Kung University Hospital, College of Medicine, National Cheng Kung University, Tainan City, Taiwan 
${ }^{12}$ Alzheimer's Disease Research Center, National Cheng Kung University Hospital, College of Medicine, National Cheng Kung University, Tainan City, Taiwan

\section{References}

1. Montero-Odasso M, Ismail Z, Livingston G. One third of dementia cases can be prevented within the next 25 years by tackling risk factors. The case "for" and "against". Alzheimers Res Ther. 2020;12(1):81.

2. Rabins PV. Developing Treatment Guidelines for Alzheimer's Disease and Other Dementias. J Clin Psychiatry. 1998;59(Suppl 11):17-9.

3. Alzheimer's A. 2016 Alzheimer's disease facts and figures. Alzheimers Dement. 2016;12(4):459-509.

4. Grossberg GT, Tong G, Burke AD, Tariot PN. Present Algorithms and Future Treatments for Alzheimer's Disease. J Alzheimers Dis. 2019;67(4):1157-71.

5. Winblad B, Kilander L, Eriksson S, Minthon L, Batsman S, Wetterholm AL, Jansson-Blixt C, Haglund A. Severe Alzheimer's Disease Study G: Donepezil in patients with severe Alzheimer's disease: double-blind, parallel-group, placebo-controlled study. Lancet. 2006;367(9516):1057-65.

6. Jann MW. Rivastigmine, a new-generation cholinesterase inhibitor for the treatment of Alzheimer's disease. Pharmacotherapy. 2000;20(1):1-12.

7. Wilkinson D, Murray J. Galantamine: a randomized, double-blind, dose comparison in patients with Alzheimer's disease. Int J Geriatr Psychiatry. 2001;16(9):852-7.

8. Tariot PN, Farlow MR, Grossberg GT, Graham SM, McDonald S, Gergel I, Memantine Study G. Memantine treatment in patients with moderate to severe Alzheimer disease already receiving donepezil: a randomized controlled trial. JAMA. 2004;291(3):317-24.

9. Wilcock G, Mobius HJ, Stoffler A group. MMM: A double-blind, placebo-controlled multicentre study of memantine in mild to moderate vascular dementia (MMM500). Int Clin Psychopharmacol. 2002;17(6):297-305.

10. Maletta GJ. Management of behavior problems in elderly patients with Alzheimer's disease and other dementias. Clin Geriatr Med. 1988;4(4):719-47.

11. Zhao L, Chen Q, Diaz Brinton R. Neuroprotective and neurotrophic efficacy of phytoestrogens in cultured hippocampal neurons. Exp Biol Med (Maywood). 2002;227(7):509-19.

12. Qian Y, Guan T, Huang M, Cao L, Li Y, Cheng H, Jin H, Yu D. Neuroprotection by the soy isoflavone, genistein, via inhibition of mitochondria-dependent apoptosis pathways and reactive oxygen induced-NF-kappaB activation in a cerebral ischemia mouse model. Neurochem Int. 2012;60(8):759-67.

13. Liu CA, Wang YJ, Na S. [Effects of soybean isoflavones on RAGE mediated signal transduction in the hippocampus of rats with Alzheimer's disease]. Zhongguo Zhong Xi Yi Jie He Za Zhi. 2012;32(6):797-800.

14. Xi YD, Li XY, Ding J, Yu HL, Ma WW, Yuan LH, Wu J, Xiao R. Soy isoflavone alleviates Abeta1-42-induced impairment of learning and memory ability through the regulation of RAGE/LRP-1 in neuronal and vascular tissue. Curr Neurovasc Res. 2013;10(2):144-56.

15. Morris MC. Nutrition and risk of dementia: overview and methodological issues. Ann N Y Acad Sci. 2016;1367(1):31-7.

16. Devi KP, Shanmuganathan B, Manayi A, Nabavi SF, Nabavi SM. Molecular and Therapeutic Targets of Genistein in Alzheimer's Disease. Mol Neurobiol. 2017;54(9):7028-41.

17. Solanki I, Parihar P, Mansuri ML, Parihar MS. Flavonoid-based therapies in the early management of neurodegenerative diseases. Adv Nutr. 2015;6(1):64-72. 
18. Guzel A, Rolz R, Nikkhah G, Kahlert UD, Maciaczyk J. A microsurgical procedure for middle cerebral artery occlusion by intraluminal monofilament insertion technique in the rat: a special emphasis on the methodology. Exp Transl Stroke Med. 2014;6:6.

19. Uluc K, Miranpuri A, Kujoth GC, Akture E, Baskaya MK. Focal cerebral ischemia model by endovascular suture occlusion of the middle cerebral artery in the rat. J Vis Exp 2011(48).

20. Loera-Valencia R, Cedazo-Minguez A, Kenigsberg PA, Page G, Duarte Al, Giusti P, Zusso M, Robert P, Frisoni GB, Cattaneo A, et al. Current and emerging avenues for Alzheimer's disease drug targets. J Intern Med.

2019;286(4):398-437.

21. Langa KM, Foster NL, Larson EB. Mixed dementia: emerging concepts and therapeutic implications. JAMA. 2004;292(23):2901-8.

22. Boutin H, LeFeuvre RA, Horai R, Asano M, Iwakura Y, Rothwell NJ. Role of IL-1alpha and IL-1beta in ischemic brain damage. J Neurosci. 2001;21(15):5528-34.

\section{Figures}

\section{Fig. 1}

a
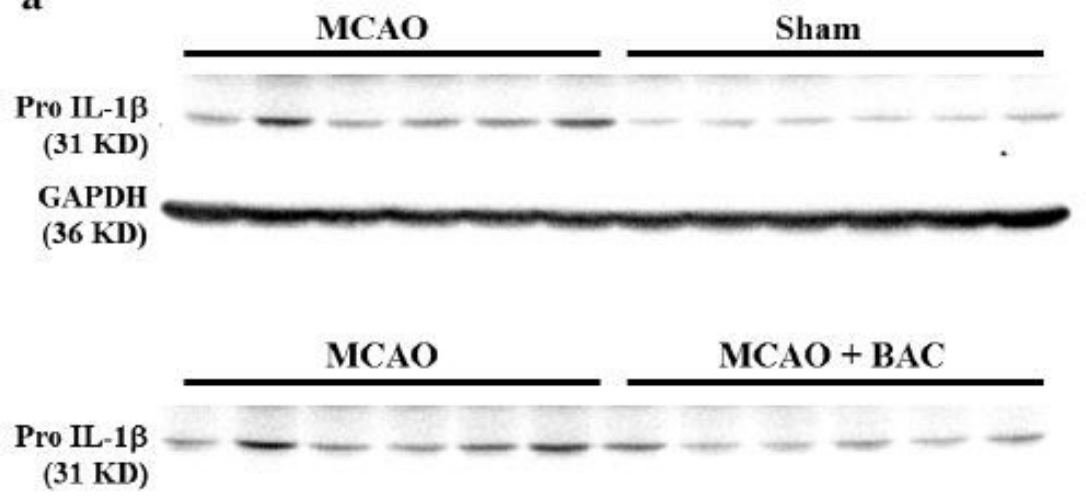

GAPDH

$(36 \mathrm{KD})$ b

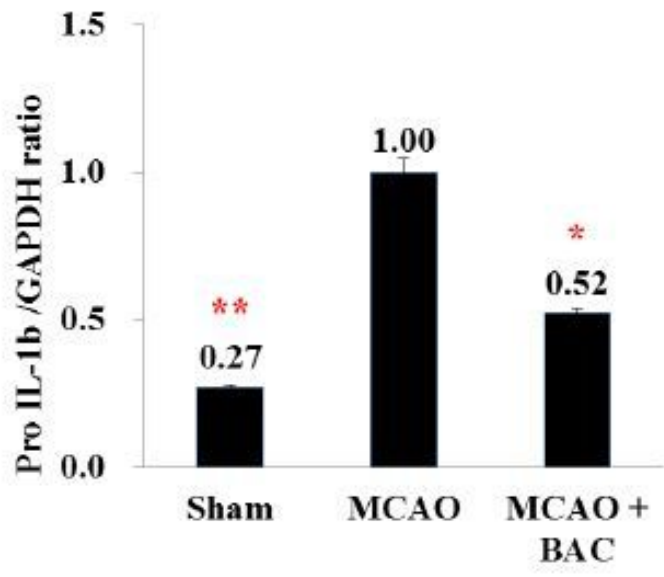

\section{Figure 1}

BAC treatment can reduce brain inflammation in $\mathrm{MCAO}$ rats. a A representative Western blot from the brains of $\mathrm{MCAO}$, sham, and MCAO treated with BAC mice (MCAO + BAC). The expression of pro-IL-1区 in the brain homogenates was detected by Western blot using antibody IL-1囚. b The signal of Western blot were quantified using ImageJ software, and GAPDH was used as the internal control. (compared with MCAO group, * $p<0.05,{ }^{\star \star} p<0.01$ ) 


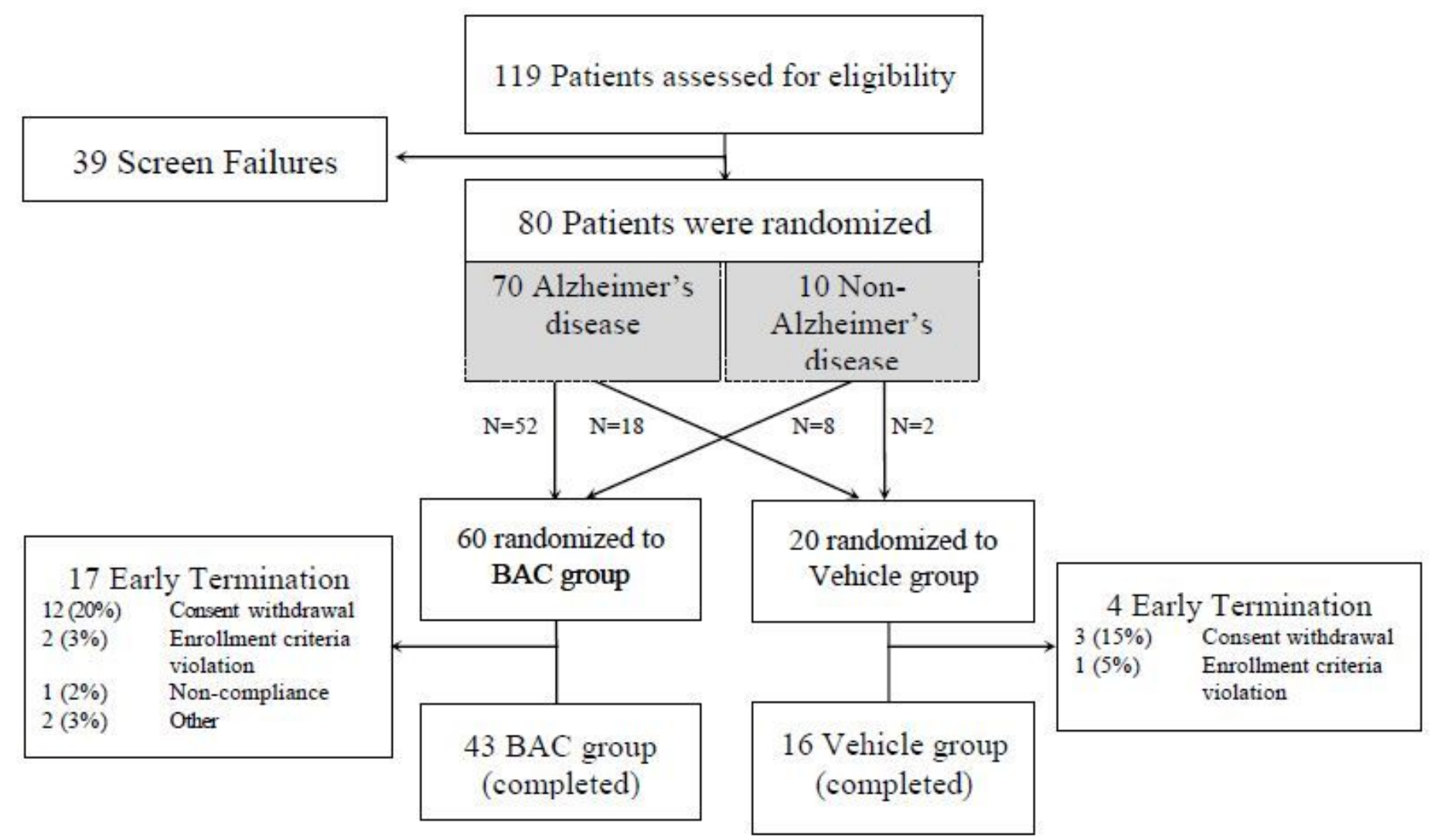

Figure 2

Patients' disposition demonstrates the number and allocation of patients from enrollment to the end of the study. The gray area indicates patients with Alzheimer's disease or non-Alzheimer's disease were randomized into the BAC group or vehicle group by a ratio of 3 to 1 . 
Fig. 3

BAC group

$90 \%$

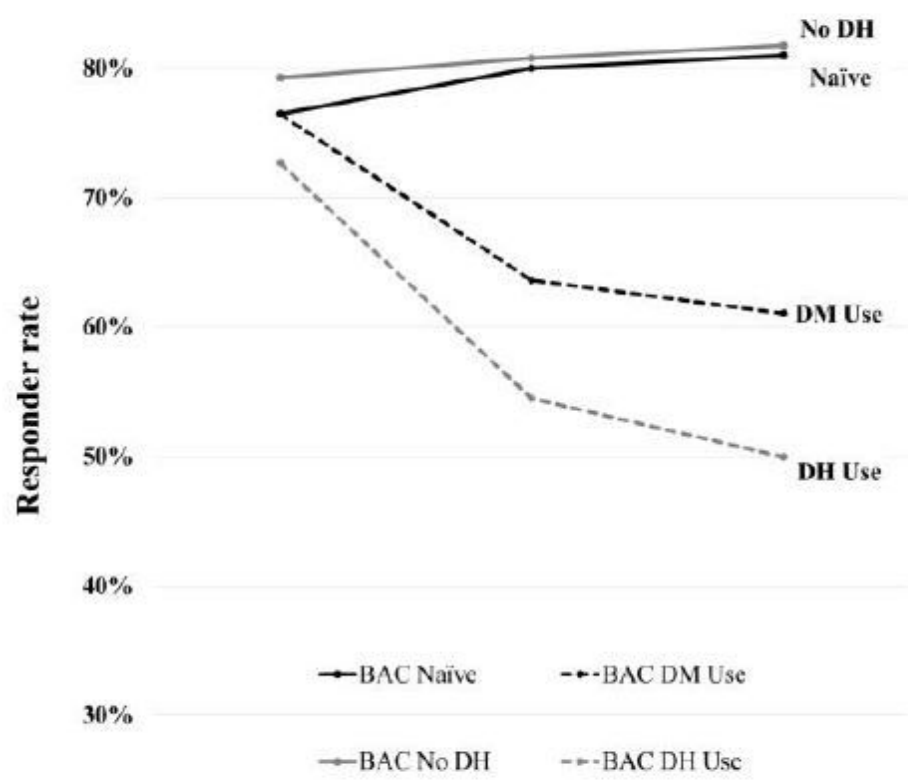

$20 \%$
$90 \%$

Vehicle group

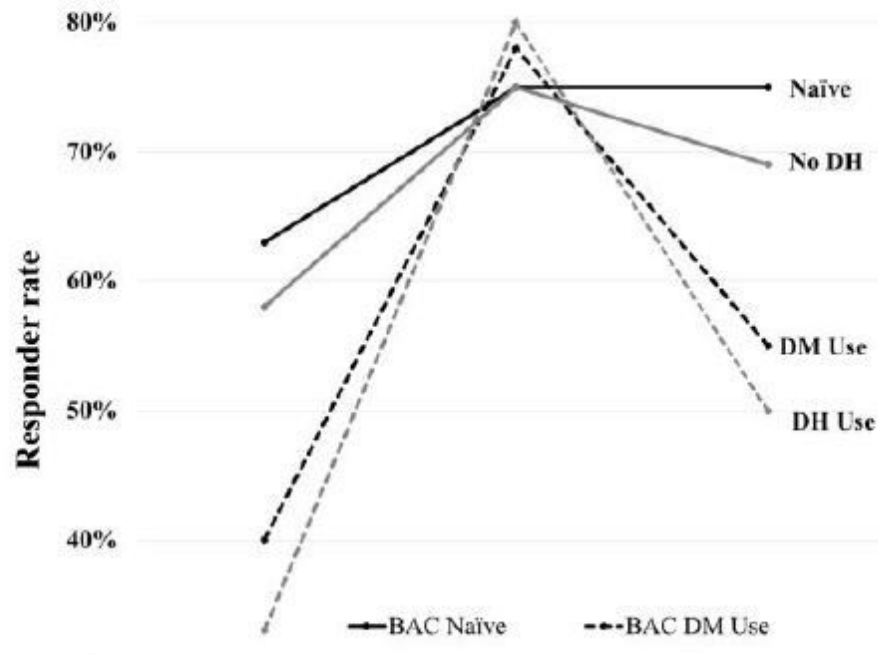

$30 \%$

$\rightarrow$ BAC No DH - -BAC DH Use

$20 \%$

Week12 - Baseline

\section{Figure 3}

The responder analysis of MMSE score in the BAC and vehicle groups. The quad-weekly change of MMSE in the percentage of responders from baseline by 4 sub-groups: naïve - no use of any dementia medication, DM Use - use of any dementia medication, No DH - no use of donepezil hydrochloride, and $\mathrm{DH}$ Use - use of DH in the BAC and vehicle group. 
Fig. 4

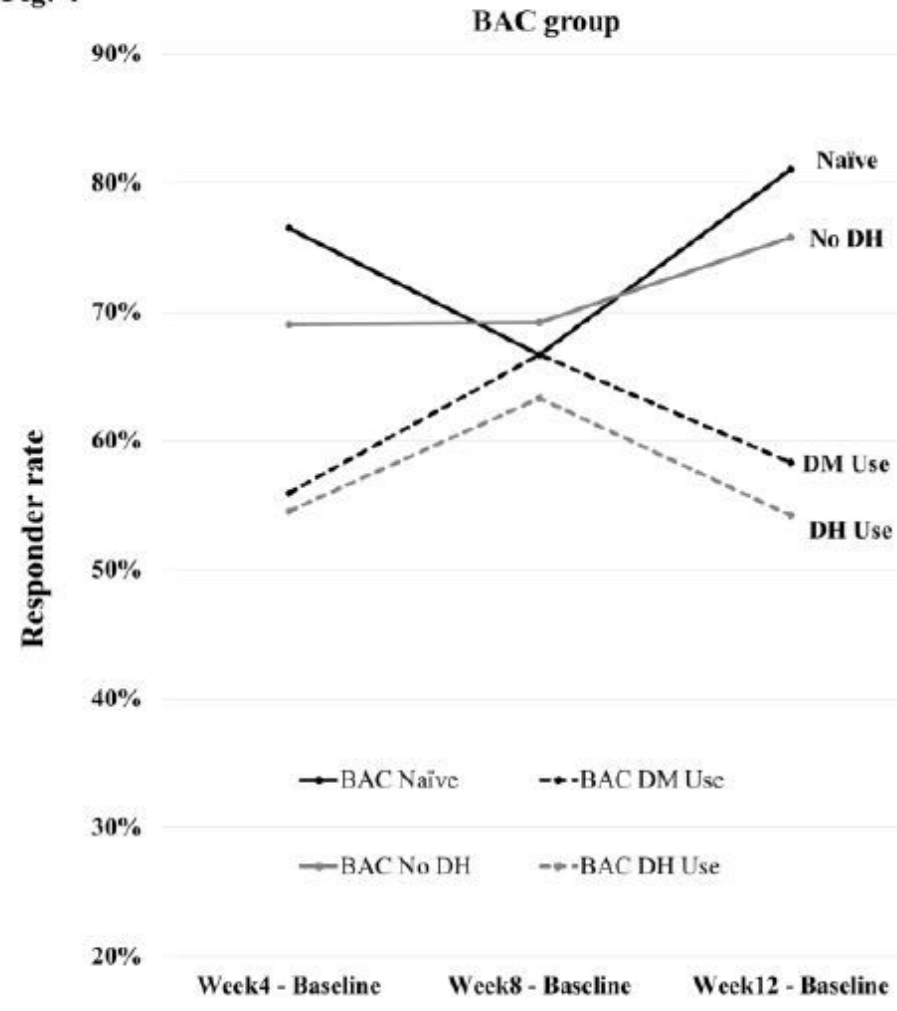

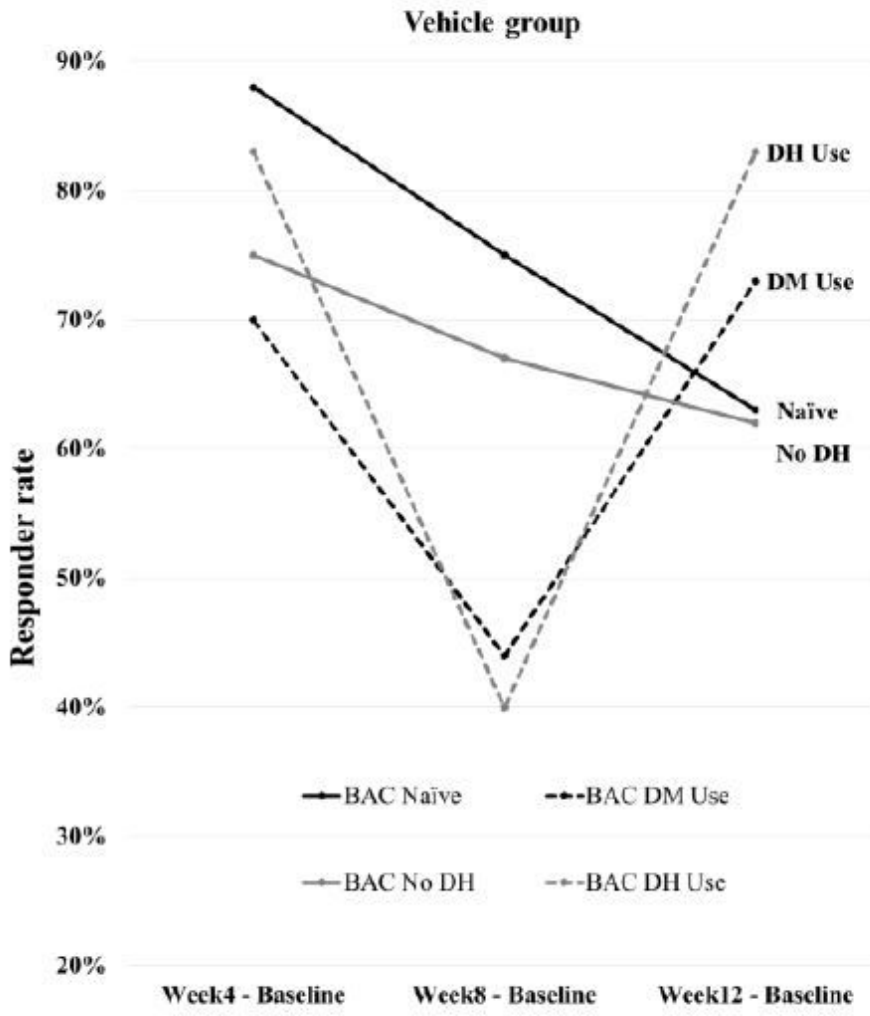

\section{Figure 4}

The responder analysis of ADAS-Cog score in the BAC and vehicle groups. The responder changes of ADAS-Cog score demonstrates the quad-weekly change in the percentage of responders from baseline by 4 sub-groups: naïve - no use of any dementia medication, DM Use - use of any dementia medication, $\mathrm{No} \mathrm{DH}$ - no use of donepezil hydrochloride, and $\mathrm{DH}$ Use - use of DH in the BAC and vehicle groups. 